ORIGINAL RESEARCH

\title{
Prophylactic Acetaminophen or Ibuprofen Result in Equivalent Acute Mountain Sickness Incidence at High Altitude: A Prospective Randomized Trial
}

\author{
Nicholas C. Kanaan, MD; Alicia L. Peterson, MD; Matiram Pun, MD; Peter S. Holck, PhD; \\ Jennifer Starling, MD; Bikash Basyal, MD; Thomas F. Freeman, MD; Jessica R. Gehner, MD; Linda Keyes, MD; \\ Dana R. Levin, MD; Catherine J. O’Leary, MD; Katherine E. Stuart, MD; Ghan B. Thapa, MD; Aditya Tiwari, MD; \\ Jared L. Velgersdyk, MD; Ken Zafren, MD; Buddha Basnyat, MD \\ From the Division of Emergency Medicine, University of Utah, (Drs Kanaan and Peterson); the Institute of Medicine, Maharajgunj, Kathmandu, \\ Nepal (Drs Pun, Basyal, Thapa, and Tiwari); the Department of Public Health, University of Hawaii (Dr Holck); the Department of Emergency \\ Medicine, Denver Health, (Dr Starling); the Department of Emergency Medicine, Augusta University, GA (Dr Freeman); the Emergency Medicine \\ Residency, Virginia Tech Carilion (Dr Gehner); the Department of Emergency Medicine, University of Colorado, Aurora, CO (Dr Keyes); the \\ Department of Aerospace Medicine, University of Texas Medical Branch (Dr Levin); the Department of Emergency Medicine, Thomas Jefferson \\ University Hospital (Dr O'Leary); the Department of Emergency Medicine, Queen's University, Kingston, ON, Canada (Dr Stuart); the \\ Department of Internal Medicine, University of North Dakota (Dr Velgersdyk); the Department of Emergency Medicine, Stanford University \\ Medical Center, Stanford, CA, and the Himalayan Rescue Association (Dr Zafren); the Oxford University Clinical Research Unit, Kathmandu, \\ Nepal, and the Himalayan Rescue Association and the Centre for Tropical Medicine and Global Health, University of Oxford, UK (Dr Basnyat).
}

Objective.-Recent trials have demonstrated the usefulness of ibuprofen in the prevention of acute mountain sickness (AMS), yet the proposed anti-inflammatory mechanism remains unconfirmed. Acetaminophen and ibuprofen were tested for AMS prevention. We hypothesized that a greater clinical effect would be seen from ibuprofen due to its anti-inflammatory effects compared with acetaminophen's mechanism of possible symptom reduction by predominantly mediating nociception in the brain.

Methods.-A double-blind, randomized trial was conducted testing acetaminophen vs ibuprofen for the prevention of AMS. A total of 332 non-Nepali participants were recruited at Pheriche (4371 m) and Dingboche $(4410 \mathrm{~m})$ on the Everest Base Camp trek. The participants were randomized to either acetaminophen $1000 \mathrm{mg}$ or ibuprofen $600 \mathrm{mg} 3$ times a day until they reached Lobuche $(4940 \mathrm{~m})$, where they were reassessed. The primary outcome was AMS incidence measured by the Lake Louise Questionnaire score.

Results.-Data from 225 participants who met inclusion criteria were analyzed. Twenty-five participants $(22.1 \%)$ in the acetaminophen group and $18(16.1 \%)$ in the ibuprofen group developed AMS $(P=.235)$. The combined AMS incidence was $19.1 \%$ (43 participants), 14 percentage points lower than the expected AMS incidence of untreated trekkers in prior studies at this location, suggesting that both interventions reduced the incidence of AMS.

Conclusions.-We found little evidence of any difference between acetaminophen and ibuprofen groups in AMS incidence. This suggests that AMS prevention may be multifactorial, affected by antiinflammatory inhibition of the arachidonic-acid pathway as well as other analgesic mechanisms that mediate nociception. Additional study is needed.

Keywords: Nepal, altitude illness, Everest, prevention, drug trials, ibuprofen, acetaminophen

Corresponding author: Buddha Basnyat, MD, Nepal International Clinic, Lal Durbar Marg-48, Kathmandu, Nepal; e-mail: buddha.basnyat@ndm.ox. ac.uk.

Submitted for publication July 2016.

Accepted for publication December 2016.

\section{Introduction}

Increasing numbers of people are visiting elevations $>2500 \mathrm{~m}$ around the globe. Individuals who ascend to high altitude and fail to acclimatize can experience 
altitude illness, including acute mountain sickness (AMS), which is characterized by a constellation of symptoms: headache, fatigue, gastrointestinal upset, dizziness, and poor sleep. ${ }^{1,2}$ These symptoms can be self-assessed and severity of illness standardized with the Lake Louise Questionnaire (LLQ). ${ }^{3}$ A diagnosis of AMS is made when the LLQ is $\geq 3$, in the presence of a headache. Along the trekking path to Everest Base Camp, the prevalence of AMS ranges from $25 \%$ to $53 \%$. ${ }^{4,5}$ AMS often is preceded by high-altitude headache (HAH), which is described as headache upon ascent to high altitude in the absence of any other AMS symptoms. Although timing of altitude illness is highly variable, it often presents within the first 24 hours of arrival to high altitude. If AMS is ignored, it can progress to dangerous and often fatal neurologic and/or pulmonary conditions, termed high-altitude cerebral edema (HACE) and high-altitude pulmonary edema. Left untreated, HACE can lead to death within 24 to 48 hours, ${ }^{6}$ thus highlighting the importance of preventing AMS. The best prevention of altitude illness is a slow ascent. ${ }^{7}$ However, proper acclimatization might be ignored or deemed impractical by mountain climbers, hikers, local pilgrims, rescue teams, or military operations.

Acetazolamide is a carbonic anhydrase inhibitor that is considered the gold standard for prophylaxis. It can help an individual acclimatize quicker and reduce the incidence of AMS. ${ }^{7,8}$ Due to its side effects, potential for causing allergic reactions, and status as a prescription medication, alternative drugs for the prevention of AMS have been sought.

The exact mechanism that causes AMS still is not understood fully. ${ }^{9}$ However, evidence points to a process in the central nervous system that increases expression of vascular endothelial growth factors, causing vasogenic edema in the brain and disruption of the blood-brain barrier. ${ }^{10,11}$ Early cerebral inflammation due to hypobaric hypoxia also has been shown to trigger an inflammatory cascade, resulting in the formation of arachidonic acid metabolites (thromboxanes, prostacyclin, and prostaglandins) as well as serotonin, histamine, and nitric oxide and bradykinin. ${ }^{12}$

The proposed mechanism for ibuprofen in AMS prophylaxis is inhibition of cyclooxygenase at the ratelimiting step in the inflammatory cascade, reducing arachidonic acid metabolites. The efficacy of dexamethasone, a steroidal anti-inflammatory medication, in the prevention and treatment of AMS and $\mathrm{HACE}^{9}$ provides a basis for the theory that the inflammatory pathway plays a major part in the pathogenesis and treatment of AMS. Three randomized, controlled trials have shown the usefulness of ibuprofen (600 mg 3 times a day [TID]) in the prevention of AMS. ${ }^{13-15}$ The response in AMS prevention to nonsteroidal anti-inflammatory drugs and steroids provides indirect evidence for a causal relationship between inflammation and altitude illness. Two studies have proven the efficacy of ibuprofen in the treatment of HAH. ${ }^{16,17}$ One of these studies ${ }^{17}$ with a small sample size found acetaminophen to be as effective as ibuprofen in the treatment of HAH. However, we found no study in the literature that investigated the efficacy of acetaminophen in the prevention of AMS.

One must also consider that headaches in AMS are multifactorial, with various chemical and mechanical factors activating a final common pathway, activation of the trigeminovascular system. The role of drugs such as acetaminophen, which primarily provides analgesia (with little anti-inflammatory effect), is unclear in the prevention of AMS. Directly comparing acetaminophen to ibuprofen for the prevention of AMS would help in better understanding these relationships and potentially the underlying pathophysiology. Therefore, this study was designed as a double-blind, randomized, prospective clinical trial comparing ibuprofen (600 mg TID) and acetaminophen (1000 mg TID) in the prevention of AMS in non-Nepali trekkers in the Himalayas. We chose this dose based on precedent, ${ }^{17}$ as well as effective pharmacologic dosing, avoiding maximum doses to minimize side effects.

\section{Methods}

A total of 332 non-Nepali volunteers aged 18 to 65 years were recruited at Pheriche $(4371 \mathrm{~m})$ and Dingboche $(4410 \mathrm{~m})$ along the Everest trekking route in the Khumbu region of Nepal. Subjects were recruited with flyers and door-to-door recruitment at the guesthouse hotels in which they stayed in Pheriche and Dingboche. Informed written consent was obtained, and participants were randomized to treatment group by a computergenerated program, prepared in advance, and held in a sealed envelope by an independent physician in the event of an emergency. Participants and researchers were blinded to the treatment group and group allocation. Each participant received 7 doses of visually matched capsules (Jolley's Compounding Pharmacy, Salt Lake City, UT) containing either ibuprofen $600 \mathrm{mg}$ or acetaminophen $1000 \mathrm{mg}$. The medication dosing and frequency was chosen to approximate prior studies, maintain visual similarities and blinding, and provide effective dosing.

Participants were excluded if upon enrollment they met criteria for AMS diagnosis (LLQ $\geq 3$ with headache), oxygen saturation $\left(\mathrm{SpO}_{2}\right)<75 \%$, or had spent $>24$ hours at altitudes $>4500 \mathrm{~m}$ in the preceding 9 days. They must not have taken acetaminophen, 


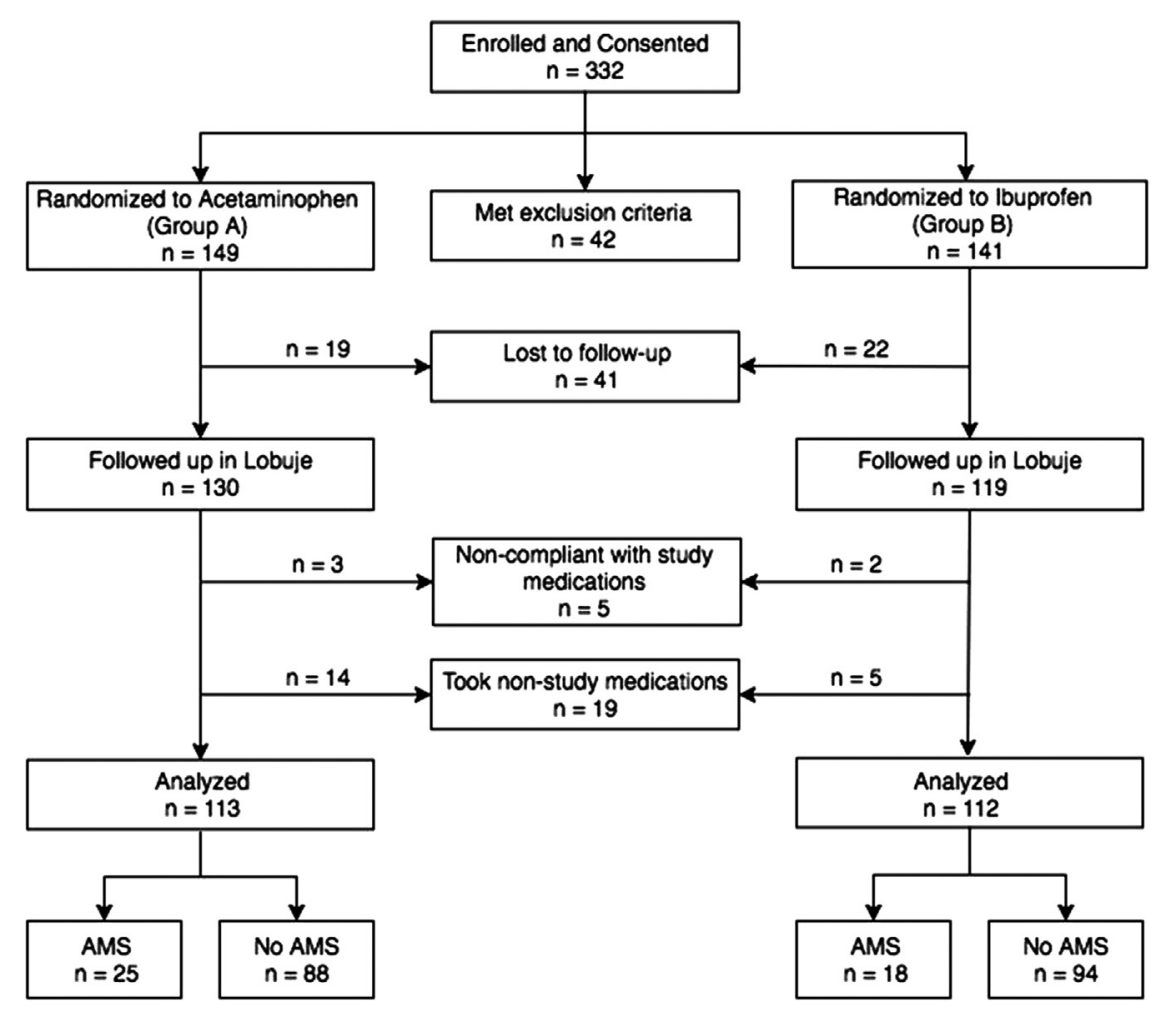

Figure 1. Study flowchart.

aspirin, nonsteroidal anti-inflammatory drugs, steroids, acetazolamide, diuretics, theophylline, or other drugs for the prevention or treatment of AMS within the previous 48 hours. Participants were screened and excluded for a history of cardiac, pulmonary, neurologic, gastrointestinal, renal, or hepatic disease. Participants who had allergies to study medications, were thought to be pregnant, or were unable to consent in English also were excluded. Nepalis were not included because most would have been well-acclimatized guides or porters who would be doing this trek for the second or third time in the season.

Participants were instructed to take their study medication 3 times daily beginning the night of enrollment (day 1). On day 2, participants acclimatized to the enrollment site and slept at the same altitude. Participants took a minimum of 3 doses at the baseline altitude before proceeding on their trek. On the third day, participants continued trekking, gaining approximately $550 \mathrm{~m}$ elevation en route to Lobuche $(4940 \mathrm{~m})$, and took their seventh and final medication dose that night. On the morning of the fourth day, participants filled out their follow-up survey on AMS symptoms prior to continuing on their trek. Participants were free to determine their rate of ascent. The baseline and repeat assessment included a self-reported $\mathrm{LLQ}^{3}, \mathrm{SpO}_{2}$ via pulse oximetry (Nonin Onyx II, Plymouth, MN) and rating of headache severity on a visual analog scale (VAS). Demographics, ascent profile, compliance, and side effects also were recorded.

A priori sample size calculation suggested 230 participants (115 per arm) would be adequate to detect a difference in AMS incidence between treatment arms $(\alpha=0.05, \beta=0.8)$ using aggregated historic data of $33 \%$ AMS incidence in placebo groups at the study site. $^{13,14,18,19}$

Analysis using both univariate (Wilcoxon rank-sum, Fisher exact, and $\chi^{2}$ tests) and multivariate (linear and logistic) methods were performed by a statistician $(\mathrm{PH})$ blinded to grouping, with significance reported at $P<.05$ using R Statistical Software (www.r-project.org). The predetermined primary end point was the incidence of AMS. Secondary end points were AMS severity (by LLQ score) and incidence and severity of headache. Ethics approval was obtained from the Nepal Health Research Council (approval 2071-3-16) and the trial was registered on www.clinicaltrial.gov (NCT02244437).

\section{Results}

A total of 332 participants consented and were enrolled and randomized to the 2 intervention groups: acetaminophen (A) and ibuprofen (B) (Figure 1). Upon enrollment, 42 were excluded by predetermined 
Table 1. Demographic data of the participants including "missed capsules" during ascent

\begin{tabular}{|c|c|c|c|}
\hline Variable & Acetaminophen $N(\%) N=113$ & Ibuprofen $N(\%) N=112$ & $P$ value \\
\hline Age $(y)$, mean \pm SD & $34.4 \pm 11.4$ & $36.8 \pm 13.1$ & .28 \\
\hline \multicolumn{4}{|l|}{ Sex } \\
\hline Female & $43(38)$ & $40(36)$ & .76 \\
\hline Male & $70(62)$ & $71(64)$ & .76 \\
\hline \multicolumn{4}{|l|}{ Ethnicity } \\
\hline White & $99(87.6)$ & $101(90.2 \%)$ & .54 \\
\hline Other & $14(12.4)$ & $11(9.8)$ & .54 \\
\hline \multicolumn{4}{|l|}{ Home altitude } \\
\hline Sea level & $80(71.4)$ & $77(69.4)$ & .05 \\
\hline$<2000 \mathrm{~m}$ & $27(24.1)$ & $34(30.6)$ & \\
\hline$>2000 \mathrm{~m}$ & $5(4.5)$ & $0(0)$ & \\
\hline History of altitude illness & $5(4.4)$ & $5(4.5)$ & .99 \\
\hline Number of missed capsules $($ mean $\pm S D)$ & $1.6 \pm 1.8$ & $1.6 \pm 1.7$ & .87 \\
\hline
\end{tabular}

criteria. Of the remaining 290 participants, 41 (14\%) were lost to follow-up and $24(8 \%)$ were noncompliant with study protocols (by taking nonstudy medications such as acetazolamide or missing $>2$ doses of study medications), leaving a population of 225 that were analyzed. The 2 groups were similar to each other with regards to demographics or questionnaire results at enrollment, aside from home altitude (Table 1). The mean number of "missed capsules" during the ascent is also included in Table 1. The ascent profile, compliance, and side effects also were recorded, and there were no significant differences except for different distribution of home altitude.

Twenty-five participants $(22.1 \%)$ in the acetaminophen group and $18(16.1 \%)$ in the ibuprofen group developed AMS during the study period; however, this difference is not statistically significant $(P=.235)$. Ibuprofen showed an observed absolute risk reduction in AMS incidence of $6.2 \%$ (95\% confidence interval [CI], 4.03-16.53\%), not significantly different from zero. AMS severity at Lobuche also was not significantly different between acetaminophen (mean LLQ $=2.2$ ) and the ibuprofen groups (mean LLQ $=1.9$ ), a reduction that also was not statistically different from zero $(P=.24)$.

In total, 43 participants $(19.1 \%)$ developed AMS. Severe AMS (LLQ >5) was diagnosed in $4(1.7 \%)$ participants in the acetaminophen group and $4(1.7 \%)$ in the ibuprofen group, without development of HACE or high-altitude pulmonary edema. No adverse events or medication side effects were reported by participants in the questionnaire, and no patients presented to the local medical clinic with any study medication-related complaints during the study or $\leq 2$ weeks after its conclusion. Outcome measures are shown for the 2 drugs in Table 2. Figure 2a reveals the LLQ score for the 2 medications in Lobuche, and Figure 2b shows the LLQ score distribution in only the participants diagnosed with AMS at Lobuche.

Although there was no difference in the severity of AMS at Lobuche in the participants using the 2 medications, the severity of headache (defined as LLQ headache subgroup score $\geq 2$ ) revealed a statistically significant reduction in the ibuprofen group $(n=3,2.5 \%)$ vs the acetaminophen group $(n=12,10.6 \%$; Table 2$)$. However, headache severity at Lobuche as measured on a VAS was similar in both groups with mean VAS $1.8 \mathrm{~mm}$ for acetaminophen and $1.2 \mathrm{~mm}$ for ibuprofen $(P=.20)$. The $\mathrm{SpO}_{2}$ change from enrollment to Lobuche was more reduced in the acetaminophen group (5.0\%) than in the ibuprofen group $(3.2 \% ; P=.01)$.

Subgroup analysis (Table 3) of each LLQ symptom score was evaluated to better understand any potential differences between interventions on individual symptoms of AMS. No statistically significant changes were noted between acetaminophen and ibuprofen groups in the distribution of LLQ symptom scores: headache, gastrointestinal upset, fatigue and weakness, dizziness and lightheadedness, or difficulty sleeping.

Multivariate analyses of outcomes adjusting for sex, age, race, prior AMS exposure, altitude of residence, and compliance provided similar conclusions to the above univariate analyses.

\section{Discussion}

The results of this study found no significant difference in the incidence and severity of AMS between prophylactic dosing of acetaminophen and ibuprofen. The combined group incidence of AMS was 19.1\%, which is notably lower than the $33 \%$ averaged historic 
Table 2. Outcome measures for acetaminophen and ibuprofen

\begin{tabular}{|c|c|c|c|c|}
\hline Outcomes & $\begin{array}{l}\text { Acetaminophen } \\
\quad(N=113)\end{array}$ & $\begin{array}{l}\text { Ibuprofen } \\
(N=112)\end{array}$ & $\begin{array}{c}95 \% \text { CI of difference } \\
\text { between groups }\end{array}$ & $P$ value \\
\hline AMS incidence at Lobuche, $\mathrm{n}(\%)^{\dagger}$ & $25(22.3)$ & $18(16.1)$ & -4.0 to 16.5 & .24 \\
\hline Mean LLQ score at Lobuche & $2.2 \pm 1.8$ & $1.9 \pm 1.6$ & -0.016 to 1.884 & .24 \\
\hline Mean VAS at Pheriche/Dingboche & $1.0 \pm 2.0$ & $0.9 \pm 1.8$ & -0.41 to 0.60 & .69 \\
\hline Mean VAS at Lobuche & $1.8 \pm 2.8$ & $1.1 \pm 1.9$ & $0.06-1.30$ & .20 \\
\hline Change in VAS & 0.7 & 0.2 & -0.11 to 1.25 & .26 \\
\hline Mean $\mathrm{SpO}_{2}$ at Pheriche/Dingboche & $87.0 \% \pm 4.0$ & $86.0 \% \pm 4.7$ & -0.15 to 2.17 & .12 \\
\hline Mean $\mathrm{SpO}_{2}$ at Lobuche & $82.0 \% \pm 5.4$ & $82.9 \% \pm 5.1$ & -2.37 to 0.57 & .28 \\
\hline Change in $\mathrm{SpO}_{2}$ & $-5.0 \%$ & $-3.2 \%$ & -3.46 to -0.30 & .01 \\
\hline Severe Headache $(\geq 2)$ at Lobuche & $12(10.6 \%)$ & $3(2.7 \%)$ & $0.02-0.14$ & .02 \\
\hline
\end{tabular}

CI, confidence interval; AMS, acute mountain sickness; LLQ, Lake Louise Questionnaire; VAS, Visual Analogue Scale; SpO ${ }_{2}$, oxygen saturation.

* Confidence intervals for continuous valued responses calculated assuming $t$-distribution.

${ }^{t} \chi^{2}$ otherwise all other analysis were performed by Wilcoxon signed-rank test.

incidence reported from placebo groups in prior studies at the same altitude and location using similar methodology and ascent rates. ${ }^{13,14,18,19}$ These results suggest that acetaminophen performs similar to ibuprofen in the prevention of AMS in partially acclimatized subjects. We hypothesize that the basis of this performance is probably not through the arachidonic acid prostaglandin pathway but through other predominantly "non-antinflammatory" mechanisms that acetaminophen may use to influence nociception in the brain. For example, axons of the trigeminal nerve (that innervate the meninges of the brain) contain pain receptors, ${ }^{20}$ which may be inhibited by acetaminophen.
Both drugs have analgesic properties, and this raises the question of whether the analgesic effect of headache reduction is the main factor. In their study Lipman et $\mathrm{a}^{15}$ provided a subgroup analysis of LLS, noting no significant difference between ibuprofen and placebo groups in the LLQ symptom scores of headache, fatigue, dizziness, and poor sleep. Similarly, in our present study of subgroup analysis (Table 3), there were no differences in the subgroup analysis. This suggests that the reduction in the incidence of AMS by acetaminophen and ibuprofen are due to a class effect applied to all variables, and not just a reduction in headache scores.
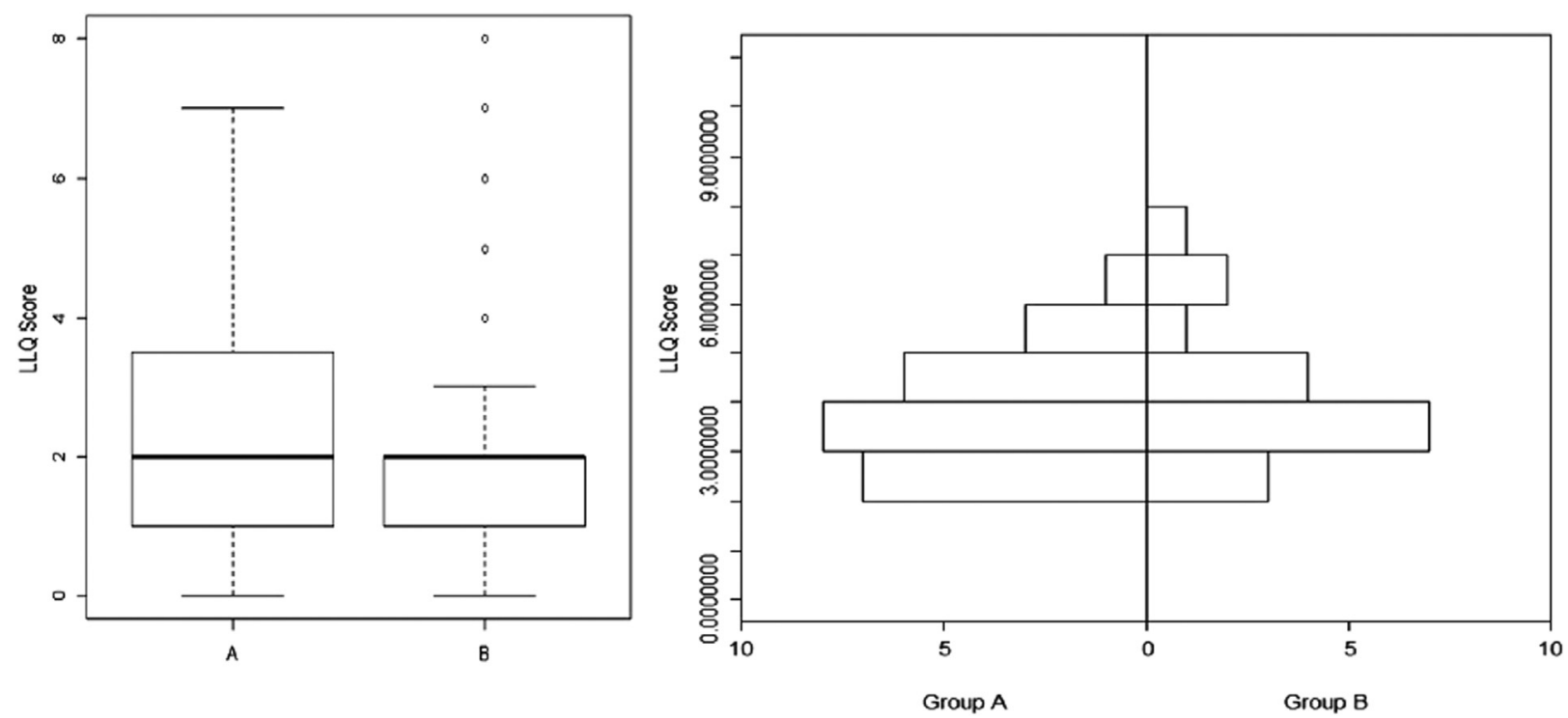

Figure 2. (A) Lake Louise Questionnaire (LLQ) score at Lobuche in acetaminophen (A) and ibuprofen (B) groups. (B) LLQ score distribution among only those diagnosed with acute mountain sickness at Lobuche. 
Table 3. Comparison of Lake Louise Criteria Questionnaire (LLQ) symptom subgroup outcomes

\begin{tabular}{lcc}
\hline LLQ Symptom Subgroup at Lobuche & OR $(95 \% \text { CI })^{*}$ & $P$ value \\
\hline Headache & $0.94(0.52-1.68)$ & .89 \\
Gastrointestinal upset & $0.49(0.19-1.17)$ & .11 \\
Fatigue/Weakness & $1.06(0.56-1.98)$ & .88 \\
Dizziness/Lightheadedness & $0.76(0.37-1.56)$ & .50 \\
Difficulty sleeping & $0.94(0.53-1.66)$ & .89 \\
\hline
\end{tabular}

${ }^{*}$ Fischer exact test.

There was a trend throughout the results showing better outcomes in the primary and secondary outcomes in the ibuprofen group compared with the acetaminophen group. The ibuprofen group had a $6.2 \%$ lower AMS incidence, a 0.3-point lower AMS severity by LLQ score, and $0.7-\mathrm{mm}$ lower headache severity by VAS by compared with the acetaminophen group, all of which were not statistically significant reductions. Although the small observed differences between the 2 treatment groups were not significantly significant, it is worthwhile to keep in mind that the study was not powered to detect small differences; thus, small, real differences may indeed exist between the 2 treatment groups. The only significant difference was ibuprofen showed a smaller reduction in oxygen saturation by $1.8 \%$ from baseline to study end point in Lobuche $(4940 \mathrm{~m})$, which also was noted in the previous study using ibuprofen. ${ }^{14}$ The clinical significance of this latter finding is limited.

Finally, although there was only a trend toward lowered headache severity in the ibuprofen group using the VAS scale, there was a significantly lower headache severity in the ibuprofen treatment group (using the LLQ headache subgroup score) suggesting that ibuprofen may be more effective than acetaminophen for the headache component of AMS diagnosis.

\section{LIMITATIONS}

Comparison of acetaminophen and ibuprofen to the standard for AMS prophylaxis, acetazolamide, may have helped better define the relative efficacies of ibuprofen and acetaminophen. Instead, with 2 interventional arms and no placebo, this study was designed to maximize the possibility of detecting a significant difference in the effects of acetaminophen and ibuprofen at altitude with the sample size available. No such differences were observed.

A placebo-control group comparison, rather than prior study historic data, would have improved our ability to evaluate the comparative efficacies of these 2 medications relative to no treatment at all. Due to logistic difficulties and resource limitations, we attempted to maximize our data collection and sample size to detect differences between acetaminophen and ibuprofen treatment groups. As such, we chose not to add a placebo arm as it would require a larger sample size than we could obtain during the trekking season; we instead used AMS incidence among placebo groups from prior studies as a comparison. Similarly, an acetazolamide study arm would have been a useful comparator, but it also was not feasible. Future studies examining inflammatory markers also could help determine the mechanism of action of these medications.

Other limitations of this study were that participants were recruited at elevation $>4000 \mathrm{~m}$ and had already began the acclimatization process during their prior days of trekking from their starting elevation of $2860 \mathrm{~m}$ at the town of Lukla. Finally, it is worth noting that although this study did not detect differences in the efficacies of ibuprofen and acetaminophen for most outcomes, a larger sample may be able to conclude that small differences similar to what were observed in this study are in fact statistically significant. That said, the sample size used was adequate to allow us to conclude that any differences, should they exist, are likely small and of questionable clinical importance.

\section{Conclusion}

This double-blind, randomized trial comparing acetaminophen $1000 \mathrm{mg}$ TID and ibuprofen $600 \mathrm{mg}$ TID for the prevention of AMS in 225 trekkers on ascent to $4940 \mathrm{~m}$ along the Everest Base Camp trekking route in Nepal detected no statistically significant difference in the incidence and severity of AMS. This finding suggests that the pathophysiology of AMS may not only be dependent on arachidonic-acid pathway and inflammation, but also other mechanisms that mediate nociception influenced by acetaminophen.

Acknowledgments: We thank the hard-working, resourceful staff of the Himalaya Rescue Association and all the participants in this study. We also thank the following field researchers: Drs Thomas Kim, 
Alexander S. Whelan, and Brett E. Sorge who worked diligently to collect field data and were integral to the study's success.

Author Contributions: Study concept and design (BB, NK, PH, MP, $\mathrm{KZ}$ ); acquisition of the data (AP, JS, TF, DL, CO, AP, BB, KS, GT, AT, JV); analysis of the data (AP, JS, TF, DL, CO, AP, BB, KS, GT, AT, JV, PH); drafting of the manuscript (NK, AP, JS, TF, PH, DL, CO, $\mathrm{KS}, \mathrm{JV}, \mathrm{BB}, \mathrm{PH}$ ); critical revision of the manuscript (NK, AP, MP, JS, $\mathrm{BB}, \mathrm{TF}, \mathrm{JG}, \mathrm{PH}, \mathrm{LK}, \mathrm{DL}, \mathrm{COL}, \mathrm{KS}, \mathrm{GT}, \mathrm{AT}, \mathrm{JV}, \mathrm{KZ}, \mathrm{BB}$ ); and approval of final manuscript (NK, AP, MP, JS, BB, TF, JG, PH, LK, DL, COL, KS, GT, AT, JV, KZ, BB).

Financial/Material Support: Partial funding for this study came from the Wellcome Trust.

Disclosures: None.

\section{References}

1. Basnyat B, Murdoch DR. High-altitude illness. Lancet. 2003;361:1967-1974.

2. Hackett PH, Roach RC. High-altitude illness. $N$ Engl J Med. 2001;345:107-114.

3. Roach RC BP, Oelz O, Hackett PH. Lake Louise AMS Scoring Consensus Committee. The Lake Louise acute mountain sickness scoring system. In: Sutton JR, Houston C, Coates G, eds. Hypoxia and Molecular Medicine. Burlington, VT: Charles S. Houston; 1993:272-274.

4. Honigman B, Theis MK, Koziol-McLain J, et al. Acute mountain sickness in a general tourist population at moderate altitudes. Ann Intern Med. 1993;118:587-592.

5. Hackett PH, Rennie D, Levine HD. The incidence, importance, and prophylaxis of acute mountain sickness. Lancet. 1976;2:1149-1155.

6. Houston CS, Dickinson J. Cerebral form of high-altitude illness. Lancet. 1975;2:758-761.

7. Luks AM, McIntosh SE, Grissom CK, et al. Wilderness Medical Society practice guidelines for the prevention and treatment of acute altitude illness: 2014 update. Wilderness Environ Med. 2014;25:S4-S14.

8. Zafren K. Prevention of high altitude illness. Travel Med Infect Dis. 2014;12:29-39.

9. Basnyat B, Tabin G. Altitude illness. In: Kasper DL, Fauci AS, Hauser SL, Longo DL, Jameson JL, Loscalzo J, eds. Harrison's Principles of Internal Medicine. 19th ed. New York, NY: McGraw Hill; 2015:476e.
10. Xu F, Severinghaus JW. Expression and response to hypoxia of vascular endothelial growth factor (VEGF) in rat and rabbit tissues. Adv Exp Med Biol. 1998;454:311-317.

11. Xu F, Severinghaus JW. Rat brain VEGF expression in alveolar hypoxia: possible role in high-altitude cerebral edema. J Appl Physiol. 1998;85:53-57.

12. Schilling L, Wahl M. Mediators of cerebral edema. Adv Exp Med Biol. 1999;474:123-141.

13. Gertsch JH, Lipman GS, Holck PS, et al. Prospective, double-blind, randomized, placebo-controlled comparison of acetazolamide versus ibuprofen for prophylaxis against high altitude headache: the Headache Evaluation at Altitude Trial (HEAT). Wilderness Environ Med. 2010;21: 236-243.

14. Gertsch JH, Corbett B, Holck PS, et al. Altitude Sickness in Climbers and Efficacy of NSAIDs Trial (ASCENT): randomized, controlled trial of ibuprofen versus placebo for prevention of altitude illness. Wilderness Environ Med. 2012;23:307-315.

15. Lipman GS, Kanaan NC, Holck PS, et al. Ibuprofen prevents altitude illness: a randomized controlled trial for prevention of altitude illness with nonsteroidal antiinflammatories. Ann Emerg Med. 2012;59:484-490.

16. Broome JR, Stoneham MD, Beeley JM, et al. High altitude headache: treatment with ibuprofen. Aviat Space Environ Med. 1994;65:19-20.

17. Harris NS, Wenzel RP, Thomas SH. High altitude headache: efficacy of acetaminophen vs ibuprofen in a randomized, controlled trial. J Emerg Med. 2003;24:383-387.

18. Gertsch JH, Basnyat B, Johnson EW, et al. Randomised, double blind, placebo controlled comparison of ginkgo biloba and acetazolamide for prevention of acute mountain sickness among Himalayan trekkers: the prevention of high altitude illness trial (PHAIT). BMJ. 2004;328:797.

19. Basnyat B, Gertsch JH, Johnson EW, et al. Efficacy of low-dose acetazolamide (125 mg BID) for the prophylaxis of acute mountain sickness: a prospective, double-blind, randomized, placebo-controlled trial. High Alt Med Biol. $2003 ; 4: 45-52$.

20. Utiger D, Eichenberger U, Bernasch D, et al. Transient minor improvement of high altitude headache by sumatriptan. High Alt Med Biol. 2002;3:387-393. 